\title{
The relationship between poor pulmonary function and irregular pulse in the elderly: findings from a nationwide cross-sectional survey in Korea
}

\section{Sun Hwa Kim}

Hanyang University Medical Center

\section{Seon Young Hwang}

Hanyang University

Jinho Shin

Hanyang University College of Medicine

Jin-Kyu Park ( $\square$ cardiohy@gmail.com )

Hanyang University College of Medicine https://orcid.org/0000-0001-7931-777X

Research article

Keywords: arrhythmia, atrial fibrillation, physical examination, pulmonary function test, pulse rate

Posted Date: April 21st, 2020

DOI: https://doi.org/10.21203/rs.3.rs-23635/v1

License: (c) (i) This work is licensed under a Creative Commons Attribution 4.0 International License.

Read Full License

Version of Record: A version of this preprint was published at Healthcare on September 1st, 2020. See the published version at https://doi.org/10.3390/healthcare8030312. 


\section{Abstract}

Background: Pulse palpation is a useful screening method for early detection of cardiac arrhythmia, which may result from reduced pulmonary function. The aim of this study is to investigate the association between reduced pulmonary function and pulse palpation finding in the elderly.

Methods: Secondary analysis was performed using the Korea National Health and Nutrition Examination Survey ( $n=2347$ subjects aged $\geq 65$ years). Pulse palpation was initially performed for 15 seconds and confirmed again for 60 seconds.

Results: The prevalence of IP was 61 (2.6\%). The mean age of subjects with IP was 73.0 (95\% Cl 71.774.3) years, and $45.8 \%$ were male. After adjustment, the lowest FVC or FEV 1 and predicted FVC $<80 \%$ remained significant risk factors for IP. A restrictive or obstructive pattern also independently predicted IP.

Conclusions: The elderly with reduced pulmonary function had a significant risk of irregular pulse, in whom careful pulse palpation may be required for early detection of arrhythmia.

\section{Background}

Cardiac arrhythmias are a group of conditions in which the heart rate is irregular, abnormally fast or slow. Some arrhythmias do not cause any symptoms and may first be discovered only incidentally during a routine examination. When the arrhythmia is chronic, some patients are unaware of its presence. Early detection and treatment of arrhythmias is therefore critical for minimizing associated complications. One of the most common arrhythmias in the elderly is atrial fibrillation (AF). This condition increases the risk of stroke five-fold and the risk of cardiac failure three-fold, leading to an increase in the mortality rate [13]. Patients usually recognize the onset of paroxysmal AF due to feeling palpitations with a sensation of a rapid heartbeat [4]. An irregular pulse (IP) can also be a sign of an abnormal heart rhythm, as it is deeply associated with AF and is a physical symptom used for screening.

An IP on physical examination is not a rare finding in primary care settings. Pulse palpation is a useful screening method for early detection of cardiac arrhythmias [5-6] and is routinely included in physical exams by clinical nurse. Opportunistic screening for early AF detection by pulse palpation in subjects 65 years of age or older is recommended by the European Society of Cardiology (ESC) guidelines [7]. This recommendation highlights the importance of appropriate and timely examination of the elderly for early detection and prevention of stroke and heart disease. Systematic population screening is useful for identifying individuals with $A F$, but the use of electrocardiograms (ECGs) to screen for AF may be expensive and time consuming [8]. The Screening for Atrial Fibrillation in the Elderly (SAFE) study concluded that opportunistic screening, such as pulse palpation and optional ECG, is the most appropriate screening method for those with IP [9]. Firzmaurice et al. also reported that pulse palpation is a cost-effective approach [10]. 
The heart and lungs are anatomically close and have highly related hemodynamics; arrhythmias (including AF) are usually found in patients suffering from chronic obstructive pulmonary disease (COPD). In cases of COPD with insufficient pulmonary function, arrhythmias are likely to occur due to the pulmonary vascular structure and the hemodynamic features of the heart. Impaired pulmonary function can trigger ectopic beats by deterioration of gas composition and pulmonary hypertension [11], which results in elevated atrial pressure and alter the electrophysiological properties of atrial tissue. These changes can trigger supraventricular tachycardia [12]. Previously, some authors have reported that impaired pulmonary function is associated with AF. A low forced expiratory volume in one second (FEV1) is also related to increased risk of AF [13]. The risk of AF was 1.8 times higher for those with an $\mathrm{FEV}_{1}$ between $60 \%$ and $80 \%$, and reduced lung function has been reported to be an independent predictor of $A F$ [14].

While subjects with reduced lung function are at greater risk for cardiac arrhythmia and may be good candidates for screening, the utility of a particular screening technique in this population is unknown. Therefore, we aim to determine whether pulse palpation is effective for screening arrhythmia in the elderly with reduced pulmonary function by examining the relationship between pulmonary function and irregular pulse (IP) using data from the Korea National Health and Nutrition Examination Survey (KNHANES).

\section{Methods}

\section{Study design}

A cross-sectional descriptive survey design using a secondary analysis of national data was adopted for this study to identify the relationship between reduced pulmonary function and IP.

\section{Setting and sample}

The present study was performed using nationally representative data from the 5th KNHANES V (20102012), a government-approved statistical survey by the Korean Centers for Disease Control and Prevention [15]. The survey was released and was available for download from the KNHANES website (https://knhanes.cdc.go.kr/) after its approval for use. The survey comprised three components: a nutrition survey for dietary assessment, a health examination survey on common chronic and cardiovascular diseases, and a health interview survey on general health conditions and health-related lifestyle [15].

For this study 4,742 subjects aged $\geq 65$ years were included from the KNHANES database and those who did not finish the health examination survey $(n=469)$ or had no spirometry data $(n=1926)$ were excluded. A total of 2,347 subjects were included in this study.

\section{Measurements}

\section{General characteristics}


A self-administered questionnaire was used to evaluate history of cigarette smoking (current smoker, exsmoker, or non-smoker), consumption of alcohol, and level of physical activity (walking, moderate, or strenuous activity), and the data was collected by interviewers. Those who smoked fewer than 100 cigarettes in their lifetime were considered as non-smokers, while those who smoked more than 100 cigarettes were classified as ex- or current smokers depending on current smoking. The frequency of alcohol consumption was divided into four categories depending on the number of drinks in the previous year (on average 1 or less per month, 2-4 times per month, 2-3 times per week, or 4 or more times per week). Alcohol intake was also classified under four categories (none, 1-4 servings per session, 5-9 servings per session, or $\geq 10$ servings per session), depending on the average drinking quantity in a single session.

Physical activity was classified into 3 categories. 'Walking' means walking regularly for 30 minutes or more in indoor or outdoor at least 5 times a week, 'moderate activity' means exercising for 30 minutes or more carrying a light item at least 3 times a week, 'strenuous activity' means activities such as climbing, running, fast biking, and carrying heavy objects for 20 minutes or more than 3 times per week.

\section{Physiological data}

Body weight, height, and waist circumference (WC) were measured by well-trained examiners. Body weight was recorded to the nearest $0.1 \mathrm{~kg}$ using a calibrated balance-beam scale (Giant-150N; Hana Co. Ltd., Seoul, Korea). A portable stadiometer (850-2060 mm; Seriter, Bismarck, ND, USA) was used to measure height to the nearest $1 \mathrm{~mm}$. Waist Circumference (WC) was measured and recorded to the nearest $0.1 \mathrm{~cm}$ in a horizontal plane at the midpoint between the iliac crest and the costal margin at the end of a normal expiration. Body mass index (BMI) was estimated as body weight (kg) divided by height squared $\left(\mathrm{m}^{2}\right)$. Blood samples were taken using a venipuncture during the health examination after an at least 8-h overnight fast. Total cholesterol (TC), serum glucose, triglycerides (TG), creatinine, and highdensity lipoprotein (HDL) cholesterol were enzymatically assessed. Blood pressure and pulse rate were measured by a nurse at a professional checkup team within the Korean Centers for Disease Control and Prevention. Blood pressure (BP) using mercury sphygmomanometers (Baumanometer; Baum, Copiague, NY) while subjects sat quietly after a five minute rest. The final BP value was calculated by taking the average value of the second and third BP measurements.

\section{Pulse palpation}

Pulse palpation measured after the subjects had rested for five minutes in a seated position, their radial pulse was initially taken for 15 seconds. Subjects with an irregular radial pulse, slow pulse (less than 15 beats) or fast pulse (more than 26 beats) the pulse was assessed again for 60 seconds. "IP" was defined as variation in the force or rhythm of impulses in radial artery during 60 seconds.

\section{Spirometry measurement}

Spirometry was performed using a dry rolling-seal spirometry device (Vmax series Sensor Medics 2130; Sensor Medics, Anaheim, CA, USA), which was operated by specially trained technicians who complied 
with the pulmonary function test (PFT) guidelines by the American Thoracic Society and European

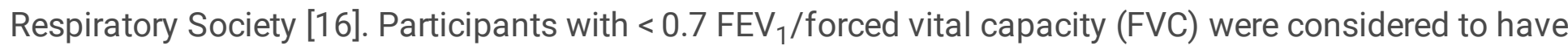
COPD. The study only used spirometry results that contained two or more acceptable curves that met the reproducibility criteria [16].

\section{Data analysis}

Statistical analyses were carried out using the Statistical Package for the Social Sciences (SPSS) program, version 18.0. The sample weights were incorporated and the analyses were adjusted for the survey's complex sample design. We weighted the survey samples in all of the analyses to generate the estimations that represented the Korean non-institutionalized civilian population. The general characteristics of the sample were calculated by considering their weighted frequency. Continuous variables are represented by mean values with confidence intervals (Cls) of $95 \%$ for after an analysis of variance (ANOVA) and categorical variables are represented by frequencies and percentages. Student's ttests and the chi-square test were employed to analyze the mean values and the categorical variables, respectively. The differences between the means were evaluated by using a one-way ANOVA. A multivariate binary logistic regression analysis was applied to assess the independent relationship of each set of spirometry data or COPD case diagnosed by a physician on IP after adjusting all the covariates such as age, sex, BMI, WC, alcohol consumption, chronic diseases, smoking status, thyroid disease, medications, and physical activity. The adjusted odds ratio (aOR) and $95 \% \mathrm{Cl}$ of IP by quartiles of $\mathrm{FVC}$ and $\mathrm{FEV}_{1}$ were estimated using the highest quartile as the reference. A two-tailed $p$-value of less than 0.05 was considered statistically significant.

\section{Results}

\section{General characteristics}

The general characteristics of all subjects are shown in Table 1. Among the 2,347 subjects, $61(2.6 \%)$ had an IP. Figure 1 shows the prevalence of IP stratified by age. The highest frequency of IP (3.9\%) was seen in those older than 80 years of age, while the prevalence of IP tended to increase with age $(p=.090)$. Smoking was not significantly different between the two groups. However, subjects with an IP had significantly more frequent alcohol consumption than those with a regular pulse (RP) $(16.9 \%$ vs. $8.3 \%$, respectively; $p=.022)$. The degrees of physical activity were similar.

\section{Physiological data comparisons}

The subjects in both groups had similar physiologic characteristics in Table 2; however, the WC of those with an IP (87.6) tended to be larger than those with a $\mathrm{RP}(84.9)(p=.065)$. There were no differences in systolic $B P$, diastolic $B P$, or pulse pressure between the two groups. However, a marginally lower heart rate was found in subjects with an IP (66.0 vs. 69.2) $(p=.085)$. None of the laboratory findings were significantly different. 


\section{Pulmonary function test and irregular pulse}

The predicted FVC (FVCp) was significantly lower (85.9\% vs. 90.6\%) ( $p=.005)$, and the prevalence of patients with FVCp $<80 \%$ was higher $(42.7 \%$ vs. $19.3 \%)(p<.001)$ in the IP group (Table 3$)$. However, there were no differences in the mean $\mathrm{FEV}_{1} / \mathrm{FVC}$ values or the prevalence of $\mathrm{FEV}_{1} / \mathrm{FVC}<0.7$ between the two groups. $\mathrm{FEV}_{1}$ was reduced (2.03 vs. 2.14$)(p=.040)$, and predicted $\mathrm{FEV}_{1}$ was lower $(88.7 \%$ vs. $93.3 \%)$ $(p=.027)$ in the IP group. Based on the interpretation of the PFT guidelines [17], more subjects in the IP group showed restrictive (22.4\% vs. $12.4 \%)$ and obstructive patterns (37.5\% vs. $29.9 \%)(p=.037)$.

\section{The effects of reduced pulmonary function on IP}

The subjects in the lowest FVC or $\mathrm{FEV}_{1}$ quartile were more likely to have an IP compared with those in the highest $\mathrm{FVC}$ or $\mathrm{FEV}_{1}$ quartile after adjustment for covariates of age, sex, BMI, WC, chronic diseases, thyroid disease, medications, smoking status, alcohol consumption, and physical activity (FVC: aOR=6.55, 95\% Cl: $1.53-28.00, p=.011 ; \mathrm{FEV}_{1}: \mathrm{aOR}=5.52,95 \% \mathrm{Cl}: 1.49-20.39, p=.011$, respectively) (Table 4). FVCp $<80 \%$ was associated with IP (aOR=2.61, 95\% Cl: $1.33-5.11, p=.005)$. Based on the PFT interpretation, subjects with a restrictive or obstructive pattern had a significantly higher risk of IP than those with normal spirometry results $(\mathrm{aOR}=2.14,95 \% \mathrm{Cl}: 1.04-4.41, p=.040)$.

\section{Discussion}

Our study found a significant correlation between reduced pulmonary function and IP in subjects aged $\geq$ 65 using nationally representative data in Korea, this finding highlights the importance of careful assessment for IP, especially in subjects with reduced pulmonary function. Pulse palpation and then optional ECG for those with an IP could help identify the associated arrhythmia, especially AF, which is the most beneficial diagnosis for the early prevention of stroke in asymptomatic and undiagnosed elderly subjects.

COPD is usually diagnosed using $\mathrm{PFT} ; \mathrm{FEV}_{1} / \mathrm{FVC}<70 \%$ confirms the presence of persistent airflow limitation and thus of COPD [18]. The worldwide prevalence of COPD is estimated to be $3-11 \%$ [19]. However, the prevalence of self-reported COPD in the present study was just $1-2 \%$. Therefore, the prevalence as assessed by PFT was higher than that determined from the patient responses on the questionnaires. This suggests that many subjects in this study had undiagnosed COPD. Yoo et al. showed a similar trend, reporting that, despite the high prevalence of COPD in Korea, the disease is actually underdiagnosed, and most COPD patients are under-treated [20].

Reduced FEV1 and obstructive pulmonary disease are associated with the incidence of AF. The Atherosclerosis Risk in Communities (ARIC) study reported that impaired pulmonary function was correlated with higher AF incidence [21], while the Malmö Preventive Project found that impaired pulmonary function was an independent predictor of AF [22]. Therefore, further evaluation of systematic $\mathrm{AF}$ screening is encouraged in at-risk populations [7]. Because the diagnosis of $\mathrm{AF}$ requires rhythm 
documentation using an ECG, we suggest that measuring pulse palpation-guided ECG diagnostic testing may help to detect $A F$ in this at-risk group. Pulse palpation is currently the evidence-based method of choice for screening for arrhythmia among individuals aged $\geq 65$ [23]. Kallmuzer et al. reported that pulse palpation at the radial artery is a simple, noninvasive, first-step screening tool to guide ECG diagnostics for cardiac arrhythmias like AF; moreover, its diagnostic accuracy is appropriate compared with continuous ECG [24]. Therefore, pulse palpation is a powerful tool for identifying subjects who require confirmatory ECG to diagnose AF. Undiagnosed AF is common, and opportunistic screening for silent AF is likely to be cost-effective in the elderly $[9,25]$. Screening of older populations yielded a prevalence of $2.3 \%$ for chronic forms of AF [26]. In the present study, the prevalence of IP was only $2.6 \%$. This finding is consistent with the low or underestimated prevalence of AF in the general population in Korea. In a healthy, asymptomatic rural Korean population, the prevalence of ECG-diagnosed AF was only $2.3 \%$ among 60- and 70-year-olds [27]. Because subjects with paroxysmal AF may be classified as having an $\mathrm{RP}$, pulse palpation may only be able to screen those with persistent or permanent AF.

Inpatient measurement of vital signs, such as pulse palpation, is usually performed by nurses. It is important that all nurses in primary or secondary care are aware of the significance of IP to detect new AF or prevent stroke in an early and timely manner. In particular, nurses working in pulmonology or cardiology departments should be well trained and cautioned not to miss IPs in patients with reduced pulmonary function and suspected AF. The present study showed that the lowest quartile of FVC or FEV ${ }_{1}$, predicted $\mathrm{FVC}<80 \%$, and a restrictive or obstructive pattern based upon spirometry interpretation were significant risk factors of IP. Therefore, clinical nurses should pay particular attention to patients with a more severely decreased $\mathrm{FVC}$ or $\mathrm{FEV}_{1}$ level or those already diagnosed with COPD or restrictive lung disease. In contrast to inpatients, who are carefully monitored by nurses, elderly community dwellers do not commonly have the opportunity for someone to check their pulse on a daily or even weekly basis. To confirm AF, outpatients should undergo further examination, such as ECG, in addition to pulse palpation. Fortunately, some authors have reported that a smartphone can be used to check for an IP at home without visiting a hospital [28-29]. This is particularly promising for elderly subjects because information technology applications will enable them to self-monitor for an IP at home without the aid of medical staff. Elderly people who are not accustomed to new technology, such as smartphones, could be assisted by family members. The appropriate application of such technology will enhance the timely detection of AF, even in elderly community members who are not hospitalized, especially those with reduced pulmonary function.

In this study, there were no statistically significant differences in the risk of IP when patients with restrictive or obstructive spirometry patterns were analyzed separately. However, when the restrictive and obstructive patterns were combined, abnormal spirometry was a significant risk factor for IP compared with a normal spirometry pattern. This finding may indicate the presence of overlap between restrictive and obstructive spirometry patterns on spirometry; 157 (36.5\%) of 474 participants with $\mathrm{FVCp}<80 \%$ were classified as having an obstructive pattern. 


\section{Limitations}

This study has a few limitations. We used a cross-sectional survey design, so our results may have been influenced by selection bias because we excluded ineligible subjects who lacked complete data. There were no available ECG data to verify specific arrhythmia diagnoses. Elderly subjects are susceptible to a wide range of different cardiac arrhythmias, many of which may be associated with radial pulse irregularities. In addition, there are no data about the period of discontinuation of smoking in ex-smokers or the exact prevalence of cardiomyopathy or thyroid disease, which might influence pulmonary function or the prevalence of arrhythmia, respectively. Lastly, COPD may be misdiagnosed using $\mathrm{FEV}_{1} / \mathrm{FVC}<0.7$ among subjects aged $\geq 65$. However, a major strength of this study was that no previous study has described the association between pulmonary function and pulse palpation in the general population. Further studies with confirmatory ECG are needed.

\section{Conclusion}

Our findings showed that reduced pulmonary function was correlated with IP in people $\geq 65$ years of age. This association suggests that the elderly with reduced pulmonary function are good candidates for early detection of cardiac arrhythmia by using pulse palpation. Healthcare providers, especially nurses, should pay close attention to assessing for the presence or absence of IPs and monitor for arrhythmias in elderly patients with reduced pulmonary function.

\section{List Of Abbreviations}

AF: atrial fibrillation; ANOVA: analysis of variance; ARIC: Atherosclerosis Risk in Communities; BMI: body mass index; BP: blood pressure; $\mathrm{Cl}$ : confidence interval; $\mathrm{COPD}$ : chronic obstructive pulmonary disease; ECG: electrocardiogram; ESC: European Society of Cardiology; $\mathrm{FEV}_{1}$ : forced expiratory volume in one second; FVC: forced vital capacity; FVCp: predicted FVC; HDL: high-density lipoprotein; IP: irregular pulse; KNHANES: Korea National Health and Nutrition Examination Survey; PFT: pulmonary function test; RP: regular pulse; SAFE: Screening for Atrial Fibrillation in the Elderly; TC: total cholesterol; TG: triglycerides; WC: waist circumference

\section{Declarations}

\section{Ethics approval and consent to participate}

The original national data was approved by the Institutional Review Board (IRB : 2012-01EXP-01-2C) of the Korean Centers for Disease Control and Prevention, and all participants were requested to submit written consent before study participation. The protocol of this study complies with the principles of the Helsinki Declaration and was approved by the IRB (2015-03-027) of H University Medical Center. 


\section{Consent for publication}

Not applicable

\section{Availability of data and materials}

The data sets used in this article can be available from the Korea Centers for Disease Control and Prevention website (https://knhanes.cdc.go.kr/knhanes/sub03/sub03_02_02.do).

\section{Competing interests}

The authors declare that they have no competing interests.

\section{Funding}

This work was supported by the National Research Foundation of Korea grant funded by the Korean government (MSIT; Ministry of Science) (No. 2019R1F1A1046443).

\section{Authors' contributions}

JKP and SHK analyzed data and made substantial contributions to research design and the acquisition, analysis, and interpretation of data. JKP, SYH and JHS helped to draft the manuscript and revise it critically for important intellectual content. All coauthors have read the final version of the manuscript.

\section{Acknowledgements}

We thank the Korean Center for Disease Control and Prevention, which carried out the KNHANES, and all the participants in the present study for their generous cooperation.

\section{Author's information}

${ }^{1}$ Stroke Unit, Hanyang University Medical Center, 222-1, Wangsimni-ro Seongdong-gu, Seoul, Korea (04763), ${ }^{2}$ College of Nursing, Hanyang University, The Second Medical Building, 222 Wangsimniro, 
Seondong-gu, Seoul, Korea (04763), ${ }^{3,4}$ Division of Cardiology, Department of Internal Medicine, College of Medicine, Hanyang University Medical Center, 222-1, Wangsimni-ro Seongdong-gu, Seoul, Korea (04763).

\section{References}

1. January CT, Wann LS, Alpert JS, Calkins H, Cigarroa JE, Cleveland JC Jr, et al. 2014 AHA/ACC/HRS guideline for the management of patients with atrial fibrillation: a report of the American College of Cardiology/American Heart Association Task Force on Practice Guidelines and the Heart Rhythm Society. J Am Coll Cardiol. 2014;64(21):e1-76. https://doi.org/10.1016/j.jacc.2014.03.022.

2. Soliman EZ, Safford MM, Muntner P, Khodneva Y, Dawood FZ, Zakai NA, et al. Atrial fibrillation and the risk of myocardial infarction. JAMA Intern Med. 2014;174(1):107-14. https://doi.org/10.1001/jamainternmed.2013.11912.

3. Mozaffarian D, Benjamin EJ, Go AS, Arnett DK, Blaha MJ, Cushman M, et al. Heart disease and stroke statistics-2015 update: a report from the American Heart Association. Circulation. 2015;131(4):e29322. https://doi.org/ 10.1161/CIR.0000000000000152.

4. Shea JB, Sears SF. Cardiology patient pages. A patient's guide to living with atrial fibrillation. Circulation. 2008;117(20):e340-43. https://doi.org/ 10.1161/CIRCULATIONAHA.108.780577.

5. Cooke G, Doust J, Sanders S. Is pulse palpation helpful in detecting atrial fibrillation? A systematic review. J Fam Pract. 2006;55(2):130-34.

6. Virtanen R, Kryssi V, Vasankari T, Salminen M, Kivela SL, Airaksinen KE. Self-detection of atrial fibrillation in an aged population: the LietoAF Study. Eur J Prev Cardiol. 2014;21(11):1437-42. https://doi.org/10.1177/2047487313494041.

7. Kirchhof P, Benussi S, Kotecha D, Ahlsson A, Atar D, Casadei B, et al. 2016 ESC Guidelines for the management of atrial fibrillation developed in collaboration with EACTS: The Task Force for the management of atrial fibrillation of the European Society of Cardiology (ESC) Developed with the special contribution of the European Heart Rhythm Association (EHRA) of the ESCEndorsed by the European Stroke Organisation (ESO). Eur Heart J. 2016;37(38):2893-962. https://doi.org/10.1093/eurheartj/ehw210.

8. Pérula-de-Torres LÁ, Martínez-Adell MÁ, González-Blanco V, Baena-Díez JM, Martín-Rioboó E, ParrasRejano JM, et al. Opportunistic detection of atrial fibrillation in subjects aged 65 years or older in primare care: a randomised clinical trial of efficacy. DOFA-AP study protocol. BMC Fam Pract. 2012;13(106):1-7. https://doi.org/10.1186/1471-2296-13-106.

9. Fitzmaurice DA, Hobbs FD, Jowett S, Mant J, Murray ET, Holder R et al. A randomised controlled trial and cost-effectiveness study of systematic screening (targeted and total population screening) versus routine practice for the detection of atrial fibrillation in people aged 65 and over. The SAFE study. Health Technol Assess. 2005;9(40):iii-iv, ix-x, 1-74.

10. Hobbs FD, Fitzmaurice DA, Mant J, Murray E, Jowett S, Bryan S, et al. Screening versus routine practice in detection of atrial fibrillation in patients aged 65 or over: cluster randomised controlled 
trial. BMJ. 2007;335(7616):383. https://doi.org/10.1136/bmj.39280.660567.55.

11. Ryu JH, Krowka MJ, Pellikka PA, Swanson KL, McGoon MD. Pulmonary hypertension in patients with interstitial lung diseases. Mayo Clin Proc. 2007;82(3):342-50. https://doi.org/10.4065/82.3.342.

12. Sideris DA, Katsadoros DP, Valianos G, Assioura A. Type of cardiac dysrhythmias in respiratory failure. Am Heart J. 1975;89(1):32-5.

13. Sin DD, Man SF. Chronic obstructive pulmonary disease: a novel risk factor for cardiovascular disease. Can J Physiol Pharmacol. 2005;83(1):8-13. https://doi.org/10.1139/y04-116.

14. Buch $P$, Friberg J, Scharling H, Lange P, Prescott E. Reduced lung function and risk of atrial fibrillation in the Copenhagen City Heart Study. Eur Respir J. 2003;21(6):1012-16.

15. Kweon S, Kim Y, Jang MJ, Kim Y, Kim K, Choi S, et al. Data resource profile: the Korea National Health and Nutrition Examination Survey (KNHANES). Int J Epidemiol. 2014;43(1):69-77. https://doi.org/10.1093/ije/dyt228.

16. Miller MR, Hankinson J, Brusasco V, Burgos F, Casaburi R, Coates A, et al. Standardisation of spirometry. Eur Respir J. 2005;26(2):319-38. https://doi.org/10.1183/09031936.05.00034805.

17. Pellegrino R, Viegi G, Brusasco V, Crapo RO, Burgos F, Casaburi R, et al. Interpretative strategies for lung function tests. Eur Respir J. 2005;26(5):948-68. https://doi.org/10.1183/09031936.05.00035205.

18. Vestbo J, Hurd SS, Agustí AG, Jones PW, Vogelmeier C, Anzueto A, et al. Global strategy for the diagnosis, management, and prevention of chronic obstructive pulmonary disease: GOLD executive summary. Am J Respir Crit Care Med. 2013;187(4):347-65. https://doi.org/10.1164/rccm.2012040596PP.

19. Buist AS, McBurnie MA, Vollmer WM, Gillespie S, Burney P, Mannino DM, et al. International variation in the prevalence of COPD (The BOLD Study): a population-based prevalence study. Lancet. 2007;370(9589):741-50. https://doi.org/10.1016/S0140-6736(07)61377-4.

20. Yoo KH, Kim YS, Sheen SS, Park JH, Hwang YI, Kim SH, et al. Prevalence of chronic obstructive pulmonary disease in Korea: the fourth Korean National Health and Nutrition Examination Survey, 2008. Respirology. 2011;16(4):659-65. https://doi.org/10.1111/j.1440-1843.2011.01951.x.

21. Li J, Agarwal SK, Alonso A, Blecker S, Chamberlain AM, London SJ, et al. Airflow obstruction, lung function, and incidence of atrial fibrillation: the Atherosclerosis Risk in Communities (ARIC) study. Circulation. 2014;129(9):971-80. https://doi.org/10.1161/CIRCULATIONAHA.113.004050.

22. Johnson LS, Juhlin T, Engstrom G, Nilsson PM. Reduced forced expiratory volume is associated with increased incidence of atrial fibrillation: the Malmo Preventive Project. Europace. 2014;16(2):182-8. https://doi.org/10.1093/europace/eut255.

23. Camm AJ, Lip GY, De Caterina R, Savelieva I, Atar D, Hohnloser SH, et al. 2012 focused update of the ESC Guidelines for the management of atrial fibrillation: an update of the 2010 ESC Guidelines for the management of atrial fibrillation. Developed with the special contribution of the European Heart Rhythm Association. Eur Heart J. 2012;33(21):2719-47. https://doi.org/10.1093/eurheartj/ehs253. 
24. Kallmünzer B, Bobinger T, Kahl N, Kopp M, Kurka N, Hilz MJ, et al. Peripheral pulse measurement after ischemic stroke: A feasibility study. Neurology. 2014;83(7):598-603. https://doi.org/10.1212/WNL.0000000000000690.

25. Davis RC, Hobbs FD, Kenkre JE, Roalfe AK, lles R, Lip GY, et al. Prevalence of atrial fibrillation in the general population and in high-risk groups: the ECHOES study. Europace. 2012;14(11):1553-9. https://doi.org/10.1093/europace/eus087.

26. Lowres N, Neubeck L, Redfern J, Freedman SB. Screening to identify unknown atrial fibrillation. A systematic review. Thromb Haemost. 2013;110(2):213-22. https://doi.org/10.1160/TH13-02-0165.

27. Park HC, Park JK, Choi SI, Kim SG, Kim MK, Choi BY, et al. Prevalence of Atrial Fibrillation and Relation to Echocardiographic Parameters in a Healthy Asymptomatic Rural Korean Population. J Korean Med Sci. 2015;30(8):1078-84. https://doi.org/10.3346/jkms.2015.30.8.1078.

28. Lee J, Reyes BA, McManus DD, Maitas O, Chon KH. Atrial fibrillation detection using an iPhone 4S. IEEE Trans Biomed Eng. 2013;60(1):203-6. https://doi.org/10.1109/TBME.2012.2208112.

29. McManus DD, Lee J, Maitas O, Esa N, Pidikiti R, Carlucci A, et al. A novel application for the detection of an irregular pulse using an iPhone $4 S$ in patients with atrial fibrillation. Heart Rhythm. 2013;10(3):315-9. https://doi.org/10.1016/j.hrthm.2012.12.001.

\section{Tables}

\section{Table 1 General Characteristics}




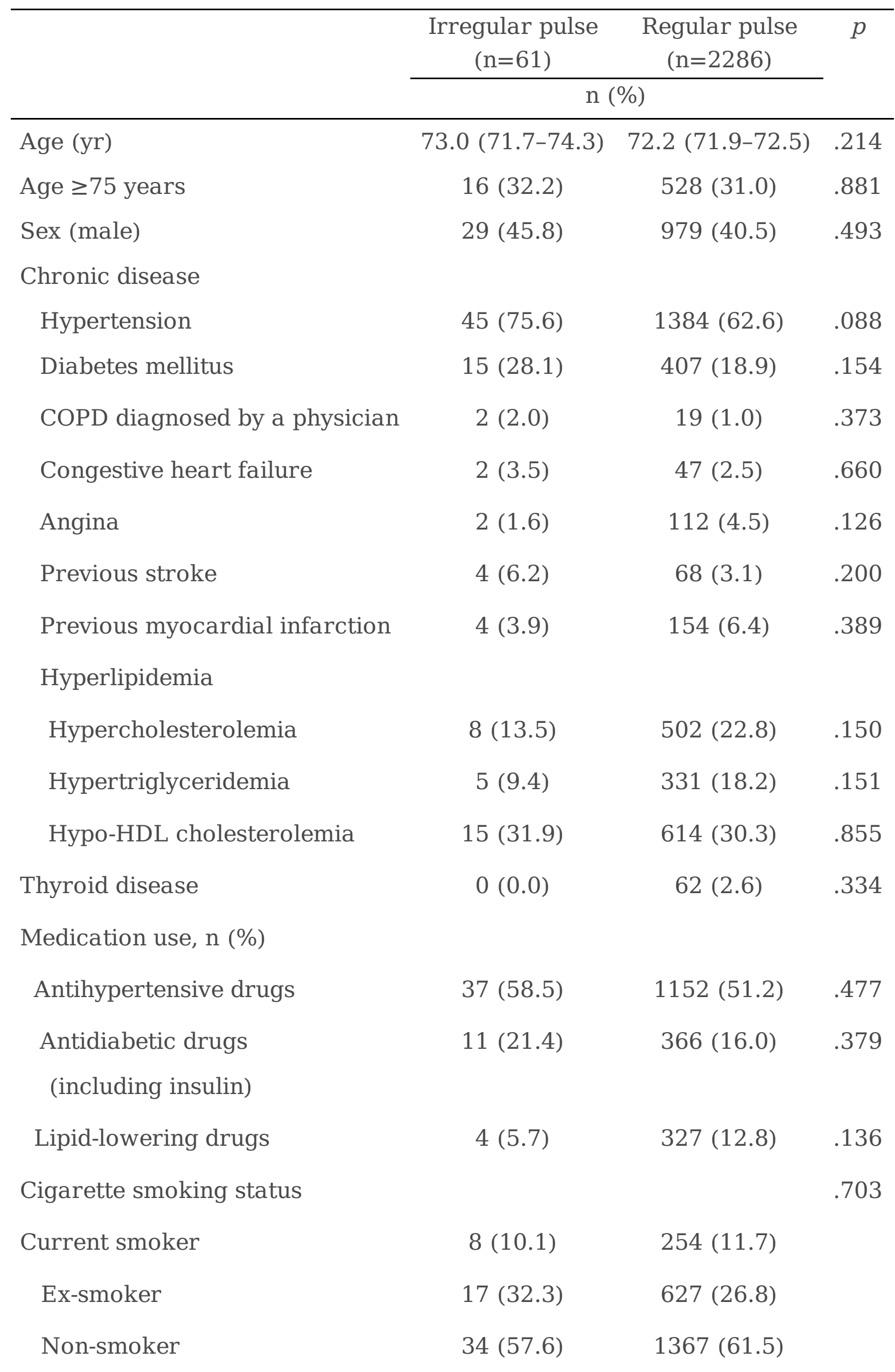




$\begin{array}{lccc}\text { Alcohol consumption frequency } & & & .022 \\ \leq 1 \text { time/month } & 44(74.6) & 1590(70.8) & \\ 2-4 \text { times/month } & 2(3.4) & 284(12.7) & \\ 2-3 \text { times/week } & 3(5.1) & 184(8.2) & \\ \geq 4 \text { times/week } & 10(16.9) & 187(8.3) & .611 \\ \text { Amount of alcohol } & 32(57.9) & 1051(49.0) & \\ \text { None } & 20(34.6) & 977(42.0) & \\ 1-4 \text { servings } & 6(5.8) & 185(7.6) & \\ 5-9 \text { servings } & 1(1.7) & 34(1.4) & \\ \geq 10 \text { servings } & & & \\ \text { Physical activity } & 21(27.6) & 885(38.1) & .202 \\ \quad \text { Walking } & 2(6.6) & 207(9.1) & .640 \\ \text { Moderate activity } & 3(4.0) & 204(8.2) & .205 \\ \quad \text { Strenuous activity } & & & \end{array}$

COPD chronic obstructive pulmonary disease, HDL high-density lipoprotein.

Continuous variables are presented as the mean (95\% confidence interval), and dichotomous variables are presented as n (\%).

\section{Table 2 Physiological Data}




\begin{tabular}{lccc}
\hline & $\begin{array}{c}\text { Irregular pulse } \\
(\mathrm{n}=61)\end{array}$ & Regular pulse $(\mathrm{n}=2286)$ & $p$ \\
\hline BMI, kg/m² & $24.8(23.9-25.8)$ & $24.2(24.0-24.4)$ & .168 \\
WC, cm & $87.6(84.7-90.5)$ & $84.9(84.2-85.5)$ & .065 \\
\hline Blood pressure, mmHg & & & \\
\hline \multicolumn{1}{c}{ Systolic } & $130.8(125.8-135.9)$ & $130.2(129.3-131.1)$ & .811 \\
\hline Diastolic & $72.1(68.5-75.8)$ & $74.3(73.8-74.9)$ & .235 \\
\hline Heart rate, bpm & $66.0(62.4-69.6)$ & $69.2(68.6-69.8)$ & .085 \\
\hline Pulse pressure, mmHg & $58.7(54.4-62.9)$ & $55.9(55.2-56.6)$ & .193 \\
\hline White blood cell count, & $6.3(5.6-7.1)$ & $6.1(6.0-6.3)$ & .616 \\
×10 ${ }^{3} / \mathrm{mm}{ }^{3}$ & & & \\
\hline Fasting glucose, mg/dL & $104.7(98.4-110.9)$ & $102.7(101.6-103.9)$ & .546 \\
\hline eGFR, mL/min/1.73 m ${ }^{2}$ & $76.3(72.7-79.9)$ & $78.9(77.7-80.1)$ & .165 \\
\hline TC, mg/dL & $185.7(174.4-197.0)$ & $193.0(190.9-195.1)$ & .208 \\
\hline HDL-C, mg/dL & $46.8(43.5-50.1)$ & $46.6(46.0-47.2)$ & .875 \\
\hline TG, mg/dL & $125.6(106.7-144.6)$ & $141.0(136.7-145.3)$ & .117 \\
\hline
\end{tabular}

BMI body mass index, WC waist circumference, eGFR estimated glomerular filtration rate, HDL-C high-density lipoprotein cholesterol, TC total cholesterol, TG triglycerides. Continuous variables are presented as the mean (95\% CI).

\section{Table 3 Spirometry Data}




\begin{tabular}{lccc}
\hline & $\begin{array}{c}\text { Irregular pulse } \\
(\mathrm{n}=61)\end{array}$ & $\begin{array}{c}\text { Regular pulse } \\
(\mathrm{n}=2286)\end{array}$ & $p$ \\
\hline FVC, L, 95\% CI & $2.84(2.67-3.01)$ & $2.95(2.91-2.99)$ & .208 \\
\hline Predicted FVC, \% & $85.9(82.6-89.2)$ & $90.6(89.9-91.2)$ & .005 \\
\hline Predicted FVC <80\%, n (\%) & $25(42.7)$ & $449(19.3)$ & $<0.001$ \\
\hline FEV $_{1} /$ FVC & $0.72(0.70-0.75)$ & $0.73(0.73-0.74)$ & .656 \\
\hline FEV $_{1} / \mathrm{FVC}<0.7, \mathrm{n}(\%)$ & $19(37.5)$ & $650(29.9)$ & .293 \\
\hline FEV, L & $2.03(1.93-2.13)$ & $2.14(2.11-2.17)$ & .040 \\
\hline Predicted FEV1, \% & $88.7(84.8-92.7)$ & $93.3(92.4-94.2)$ & .027 \\
\hline Interpretation, n (\%) & & & .037 \\
\hline Normal & $27(40.1)$ & $1334(57.7)$ & \\
\hline Restrictive & $15(22.4)$ & $302(12.4)$ & \\
\hline Obstructive & $19(37.5)$ & $650(29.9)$ & \\
\hline
\end{tabular}

FVC forced vital capacity, $\mathrm{FEV}_{1}$ forced expiratory volume in $1 \mathrm{~s}$.

Table 4 Logistic Regression analysis of Pulmonary Function for Irregular Pulse 


\begin{tabular}{|c|c|c|c|}
\hline & Adjusted $\mathrm{OR}^{\dagger}$ & $95 \% \mathrm{CI}$ & $p$ \\
\hline \multicolumn{4}{|l|}{ FVC, L } \\
\hline Quartile 1 (FVC <2.44) & 6.55 & $1.53-28.00$ & .011 \\
\hline Quartile 2 (FVC 2.44-2.89) & 2.22 & $0.50-9.88$ & .294 \\
\hline Quartile 3 (FVC 2.90-3.52) & 3.00 & $0.96-9.32$ & .058 \\
\hline Quartile 4 (FVC $\geq 3.53$ ) & 1 & & \\
\hline Predicted FVC, \% & 0.98 & $0.96-1.00$ & .052 \\
\hline Predicted FVC < $<0 \%$ & 2.61 & $1.33-5.11$ & .005 \\
\hline \multicolumn{4}{|l|}{ FEV1, L } \\
\hline Quartile 1 (FEV1 <1.83) & 5.52 & $1.49-20.39$ & .011 \\
\hline Quartile 2 (FEV1 1.83-2.14) & 4.80 & $1.26-18.29$ & .022 \\
\hline Quartile 3 (FEV1 2.14-2.54) & 3.06 & $1.06-8.89$ & .040 \\
\hline Quartile 4 (FEV1 $\geq 2.54$ ) & 1 & & \\
\hline Predicted FEV1, \% & 0.99 & $0.97-1.00$ & .065 \\
\hline \multicolumn{4}{|l|}{ Interpretation } \\
\hline Normal & 1 & & \\
\hline Restrictive & 2.45 & $0.95-6.33$ & .065 \\
\hline Obstructive & 1.95 & $0.92-4.12$ & .079 \\
\hline Restrictive or obstructive & 2.14 & $1.04-4.41$ & .040 \\
\hline COPD diagnosed by a physician & 1.18 & $0.12-11.73$ & .885 \\
\hline
\end{tabular}

COPD chronic obstructive pulmonary disease, FVC forced vital capacity, FEV forced expiratory volume in $1 \mathrm{~s}$.

${ }^{\dagger}$ Odds ratios were obtained from a logistic regression model adjusted for age, sex, BMI, waist circumference, chronic diseases, thyroid disease, medications, smoking status, alcohol consumption, and physical activity.

\section{Figures}




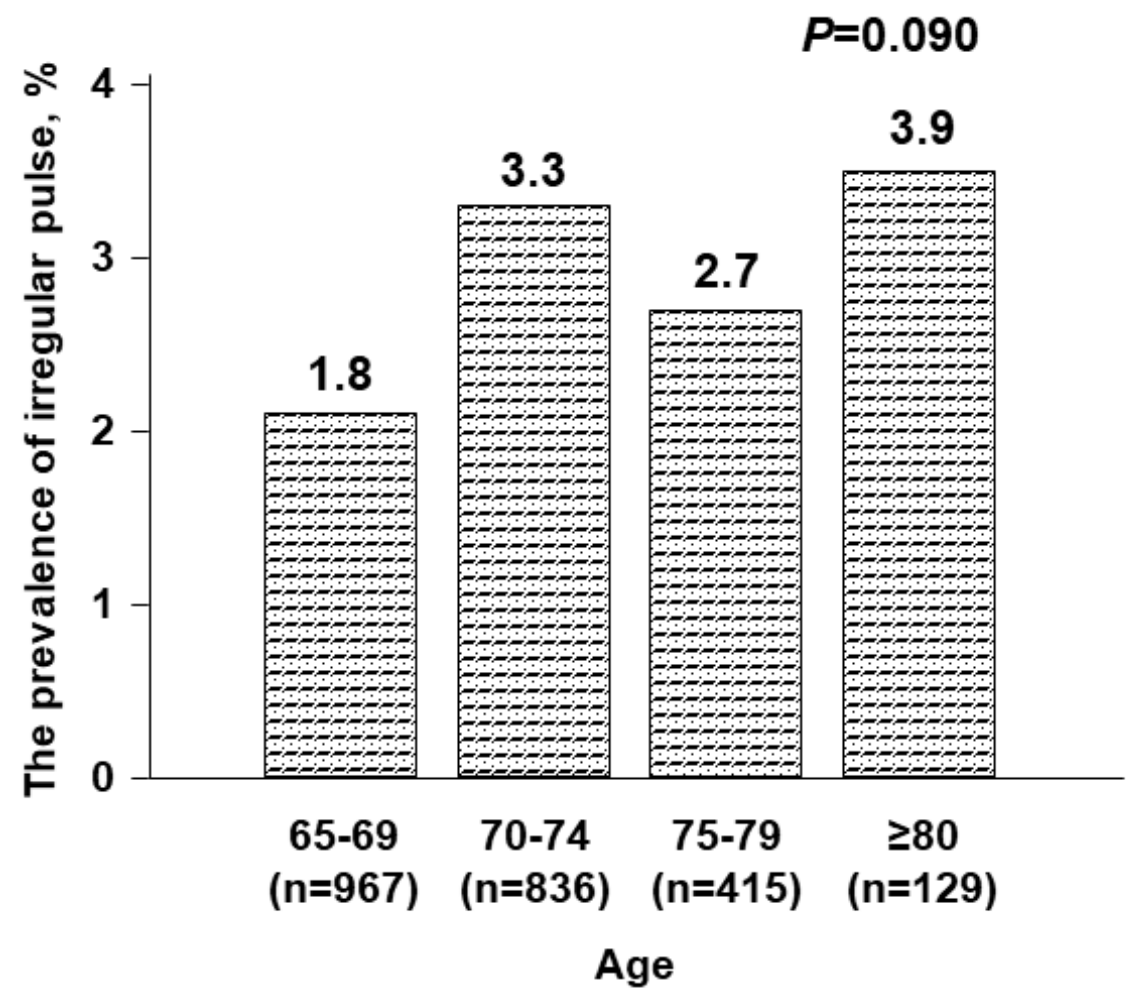

\section{Figure 1}

Figure 1

Prevalence of irregular pulse in the general population $\geq 65$ years old. 